\title{
Desempenho de alevinos de tilápia do Nilo alimentados com folha de mandioca desidratada na dieta
}

\author{
[Performance of Nile tilapia fingerlings fed dehydrated cassava leaf in the diet] \\ E.L. Santos ${ }^{1}$, K.S. Bezerra ${ }^{2}$, E.C.S. Soares ${ }^{1}$, T.J. Silva ${ }^{1}$, C.H.L.H. Ferreira ${ }^{1}$, \\ C.C.S. Santos ${ }^{1}$, C.F. Silva ${ }^{1}$ \\ ${ }^{1}$ Universidade Federal de Alagoas, Centro de Ciências Agrárias - UFAL - Rio Largo, AL \\ ${ }^{2}$ Universidade Federal do Vale do São Francisco - UNIVASF - Petrolina, PE
}

\begin{abstract}
RESUMO
O objetivo foi avaliar o crescimento e a viabilidade econômica de alevinos de tilápia do Nilo (Oreochromis niloticus), alimentados com diferentes níveis de farinha da folha de mandioca desidratada na ração (FFMD). Foram utilizados 80 alevinos distribuídos em 20 recipientes de 18L, constando de sistema de recirculação de água, durante um período de 51 dias. Cem exemplares, alimentados três vezes ao dia até a saciedade, foram distribuídos aleatoriamente em um delineamento experimental inteiramente casualizado, constituindo-se de 4 tratamentos (0, 5, 10 e 15\% de adição da FFMD na ração) e 5 repetições cada. Os peixes alimentados com 10\% de FFMD apresentaram melhor homogeneidade no crescimento. Entretanto, os indivíduos alimentados com inclusão de 5\% de FFMD apresentaram homogeneidade de crescimento similar aos alimentados com $10 \%$. Além disso, os peixes alimentados com 5\% de FFMD obtiveram melhor desempenho zootécnico e viabilidade econômica da ração. Dessa maneira, recomendase a utilização de $5 \%$ da farinha de folha de mandioca desidratada em rações para tilápia do Nilo.
\end{abstract}

Palavras-chave: alimentos alternativos, desempenho, Oreochromis niloticus

\begin{abstract}
The aim was to evaluate the growth and the economic viability of the Nile tilapia (Oreochromis niloticus) fed with different levels of dehydrated cassava leaf flour in the diet (FFMD). 80 fingerlings were distributed into $2018 \mathrm{~L}$ containers, consisting of a water recirculation system over a period of 51 days. 100 copies fed three times a day to satiation were randomly assigned in a completely randomized design, constituting 4 treatments $(0 \%, 5 \%, 10 \%$ and $15 \%$ addition of FFMD in diet) with 5 repetitions each. The fishes fed with 10\% FFMD had better homogeneity in growth. However, individuals fed with inclusion of $5 \%$ FFMD homogeneity grew similarly to those fed with 10\% FFMD. In addition, the fish from the 5\% had better growth performance and economic viability of the feed. Thus, it is recommended to use $5 \%$ of dehydrated cassava leaf meal in diets for Nile tilapia.
\end{abstract}

Keywords: alternative foods, performance, Oreochromis niloticus

\section{INTRODUÇÃO}

A piscicultura é uma das atividades produtivas que mais se desenvolvem no Brasil, principalmente devido a certos fatores peculiares que a torna promissora e sustentável, como destacadamente as favoráveis condições climáticas e a abundância de recursos hídricos. Nesse contexto, a região Nordeste se destaca,

Recebido em 20 de fevereiro de 2015

Aceito em 27 de agosto de 2015

E-mail: elton@zootecnista.com.br pois apresenta espécies de grande potencial de cultivo e versatilidade, adaptadas ao ambiente tropical (Santos et al., 2013). A tilápia do Nilo (Oreochromis niloticus) é uma espécie de peixe bastante versátil na piscicultura, pois se adapta a qualquer tipo de cultivo, sem nenhuma restrição à tecnologia que seja necessária empregar. É a segunda espécie de peixe cultivada em água doce, e a mais produzida no Brasil (Brasil, 2011). 
A procura por alimentos alternativos para rações de peixes tem encontrado nos subprodutos da mandioca uma possibilidade para substituir cereais tradicionais. Esses subprodutos são excelentes alternativas para baratear os custos com a alimentação animal, que representam cerca de $70 \%$ dos custos de produção na piscicultura (Santos et al., 2009a). Entre esses subprodutos, destaca-se a farinha da folha da mandioca desidratada, possuindo alto valor nutritivo, além de excelente aceitabilidade pelos animais.

A farinha da folha de mandioca desidratada é uma fonte de proteína de baixo custo, produzida a partir de folhas e hastes da mandioca descartadas pelos produtores. Esse ingrediente quando comparado a outros componentes usualmente utilizados nas rações, como o farelo de soja, pode suprir as exigências proteicas dos peixes e reduzir o custo da ração.

A realização de estudos direcionados à elaboração de rações (Boscolo et al., 2002; Lacerda et al., 2005) contendo esse tubercúlo desidratado em seu processamento pode indicar dietas nutricionalmente completas, para uso com alevinos de tilápia do Nilo, podendo reduzir os custos de produção e do crescimento heterogêneo dos lotes de animais na tilapicultura.

Dessa forma, o presente trabalho tem como objetivo avaliar o desempenho zootécnico e o crescimento da tilápia do Nilo alimentada com folha de mandioca desidratada, analisando a viabilidade econômica da inclusão desse ingrediente na dieta.

\section{MATERIAL E MÉTODOS}

O estudo foi realizado em 20 aquários experimentais com volume útil de $18 \mathrm{~L}$, dotados de um sistema de recirculação de água com filttro biológico. Oitenta alevinos de tilápia do Nilo, machos revertidos, com média de peso inicial de $3,0 \pm 0,5 \mathrm{~g}$ foram divididos em grupos de quatro e distribuídos aleatoriamente em cada unidade experimental, onde permaneceram por um período de 51 dias.

As quatro dietas foram formuladas para obterem valores nutricionais similares (Tab. 1), dentro das exigências para a espécie, de acordo com o NRC (1993). Para o processamento da ração, todos os ingredientes foram moídos, homogeneizados, umedecidos com água a $65^{\circ} \mathrm{C}$, peletizados $\mathrm{e}$ desidratados em estufa de ventilação forçada a $65^{\circ} \mathrm{C}$. Após esse procedimento, os péletes foram padronizados e separados de acordo com os diâmetros compatíveis ao tamanho da boca dos animais. Esta adequação ocorreu sucessivamente, à medida que os indivíduos cresciam.

Foram utilizadas concentrações de 0, 5, 10 e $15 \%$ de farinha de folha de mandioca desidratada (FFMD) nas rações. $\mathrm{O}$ delineamento experimental consistiu em quatro tratamentos e cinco repetições, totalizando 20 parcelas experimentais distribuídas ao acaso.

Após o período de aclimatação (sete dias), a oferta de alimento foi realizada até a aparente saciedade do animal, três vezes ao dia $(8 \mathrm{~h}, 12 \mathrm{~h}$ e 16h), de forma manual. Inicialmente, os alevinos foram pesados em balança eletrônica de precisão 0,1g; passados 51 dias, estes foram submetidos a jejum de $24 \mathrm{~h}$, com posterior anestesiamento com óleo de cravo na água $(250 \mathrm{mg} / \mathrm{L})$ e abate por choque térmico a $0^{\circ} \mathrm{C}$ (água com gelo), até cessarem os sinais vitais para posterior biometria final.

Os indicadores de qualidade de água: $\mathrm{pH}$, oxigênio e temperatura foram monitorados diariamente, em dois horários (7h45min e 17h00), por meio de sonda multiparamétrica da marca HANNA Instruments, modelo 9828, Woonsocket, USA, a uma profundidade de aproximadamente $20 \mathrm{~cm}$ abaixo da lâmina d'água. A amônia total $\left(\mathrm{NH}_{3}+\mathrm{NH}_{4}\right)$ foi mensurada, em avaliações quinzenais, com o auxílio de espectrofotômetro da marca HANNA Instruments, modelo HI 83203, Bélgica, utilizando os reagentes de modelo HI93700-01. $\mathrm{O}$ nitrato $\left(\mathrm{NO}_{3}^{-}\right)$e o fósforo (P) dos efluentes foram analisados com esse mesmo equipamento, utilizando os reagentes HI93705-01 para o nitrito.

A heterogeneidade dos peixes foi analisada pelo cálculo de crescimento heterogêneo (Chet), através do coeficiente de variação do peso do animal $(\mathrm{CV}=$ desvio padrão/média do peso $\mathrm{x}$ 100). 
Tabela 1. Composição percentual das dietas experimentais

\begin{tabular}{|c|c|c|c|c|}
\hline \multirow{2}{*}{ Ingredientes (\%) } & \multicolumn{4}{|c|}{ Tratamentos } \\
\hline & $0 \%{ }^{1}$ & $5 \%^{2}$ & $10 \%^{3}$ & $15 \%^{4}$ \\
\hline Farelo de soja $45 \%$ & 54,10 & 50,60 & 48,18 & 44,37 \\
\hline Milho grão & 22,56 & 21,30 & 16,22 & 17,56 \\
\hline Farelo de trigo & 12,00 & 12,00 & 11,91 & 12,00 \\
\hline Farinha de Peixe 55\% & 5,99 & 6,00 & 6,00 & 6,00 \\
\hline Óleo de soja & 2,65 & 2,40 & 2,98 & 2,26 \\
\hline Premix vitamínico/ mineral ${ }^{5}$ & 1,00 & 1,00 & 1,00 & 1,00 \\
\hline Fosfato bicálcico & 0,63 & 0,67 & 0,72 & 0,75 \\
\hline Sal comum & 0,50 & 0,50 & 0,50 & 0,50 \\
\hline DL-metionina & 0,31 & 0,50 & 0,45 & 0,50 \\
\hline Calcário & 0,26 & 0,03 & 2,04 & 0,06 \\
\hline Folha de mandioca (FFMD) & 0,00 & 5,00 & 10,00 & 15,00 \\
\hline TOTAL & 100,00 & 100,00 & 100,00 & 100,00 \\
\hline \multicolumn{5}{|l|}{ NUTRIENTES $^{6}$} \\
\hline ED (kcal/kg) & 3.071 & 3.066 & 3.057 & 3.049 \\
\hline PB - Proteína bruta (\%) & 32,00 & 32,00 & 32,00 & 32,00 \\
\hline FB - Fibra Bruta (\%) & 4,81 & 5,40 & 5,87 & 6,60 \\
\hline EE - Extrato etéreo (\%) & 5,01 & 5,00 & 5,59 & 5,31 \\
\hline Metionina +cistina (\%) & 1,14 & 1,21 & 1,20 & 1,20 \\
\hline Lisina $(\%)$ & 1,96 & 1,95 & 1,88 & 1,86 \\
\hline Triptofano (\%) & 0,34 & 0,33 & 0,33 & 0,33 \\
\hline Valina (\%) & 1,49 & 1,48 & 1,43 & 1,41 \\
\hline Treonina (\%) & 1,28 & 1,27 & 1,27 & 1,27 \\
\hline Arginina (\%) & 2,14 & 2,13 & 2,05 & 2,03 \\
\hline Leucina (\%) & 2,17 & 2,14 & 2,14 & 2,13 \\
\hline Fenilalanina + tirosina $(\%)$ & 2,23 & 2,22 & 2,13 & 2,12 \\
\hline Histidina (\%) & 0,79 & 0,78 & 0,75 & 0,73 \\
\hline Treonina (\%) & 1,26 & 1,23 & 1,22 & 1,22 \\
\hline Isoleucina (\%) & 1,43 & 1,38 & 1,33 & 1,32 \\
\hline Cálcio (\%) & 0,82 & 0,79 & 0,87 & 0,77 \\
\hline Fósforo disponível (\%) & 0,51 & 0,50 & 0,51 & 0,50 \\
\hline
\end{tabular}

${ }^{1}-0 \%$ de inclusão da farinha da folha de mandioca desidratada.

${ }^{2}-5 \%$ de inclusão da farinha da folha de mandioca desidratada.

${ }^{3}-10 \%$ de inclusão da farinha da folha de mandioca desidratada.

${ }^{4}-15 \%$ de inclusão da farinha da folha de mandioca desidratada.

5 - Niveis de garantia por kg do produto: vit. $\mathrm{A}=900.000 \mathrm{UI}$; vit. D3 = 50.000 UI; vit. $\mathrm{E}=6.000 \mathrm{mg}$; vit. K3 = $.1200 \mathrm{mg}$; vit. B1 = 2.400mg; vit. B2 = 2.400mg; vit. $\mathrm{B} 6=2.000 \mathrm{mg}$; vit. $\mathrm{B} 12=4.800 \mathrm{mg} ;$ folic acid = $1.200 \mathrm{mg}$; calcium pantothenate $=12.000 \mathrm{mg}$; vit. $\mathrm{C}=24.000 \mathrm{mg}$; biotina $=6,0 \mathrm{mg}$; choline $=65.000 \mathrm{mg} ;$ niacin $=24.000 \mathrm{mg} ; \mathrm{Fe}$ $=10.000 \mathrm{mg} ; \mathrm{Cu}=600 \mathrm{mg} ; \mathrm{Mn}=4.000 \mathrm{mg} ; \mathrm{Zn}=6.000 \mathrm{mg} ; \mathrm{I}=20 \mathrm{mg} ; \mathrm{Co}=2,0 \mathrm{mg}$ e $\mathrm{Se}=25 \mathrm{mg}$.

6 - De acordo com Rostagno (2005).

Em relação à viabilidade econômica da utilização do FFMD - farinha de folha de mandioca desidratada - nas rações, foi determinado o custo médio em ração por quilo de peso vivo (Yi), durante $o$ período experimental, conforme recomendações de Silva et al. (2008), seguindo as fórmulas: 1. CMR $(\mathrm{R} \$ / \mathrm{kg})=\mathrm{Qi} \mathrm{X} \mathrm{Pi} / \mathrm{Gi}$, em que $\mathrm{CMR}=$ custo da ração por $\mathrm{kg}$ de peso vivo ganho no i-enésimo tratamento; Qi = quantidade de ração consumida no i-enésimo tratamento; $\mathrm{Pi}$ $=$ preço por $\mathrm{kg}$ da ração utilizada no i-enésimo tratamento; $\mathrm{Gi}=$ ganho de peso do i-enésimo tratamento; 2 IEE $(\%)=$ MCe/CTei X 100 e IC $(\%)=$ CTei/MCe X 100, em que MCe = menor custo da ração por $\mathrm{kg}$ ganho observado entre os tratamentos; Ctei = custo do tratamento $\mathrm{i}$ considerado.

Os dados foram ordenados em tabela do ExcelMicrosoft $^{\circledR}$ e analisados de forma descritiva. Além disso, realizou-se análise de variância (ANOVA) à probabilidade de $5 \%$ e o teste de 
comparação de médias de Tukey e análise de regressão no nível de 5\% de probabilidade.

Esta pesquisa está de acordo com os princípios éticos em pesquisa com animais e foi aprovada pelo Comitê de Ética no Uso de Animais da Universidade Federal de Alagoas, Brasil (Protocolo no: 0039 /14 - CEUA/UFAL).

\section{RESULTADOS E DISCUSSÃO}

Os parâmetros de qualidade da água foram monitorados diariamente; as concentrações de oxigênio dissolvido na água $(9,58 \pm 1,03 \mathrm{mg} / \mathrm{L})$, temperatura $\left(25,99 \pm 0,67^{\circ} \mathrm{C}\right), \mathrm{pH}(6,99 \pm 0,14)$, amônia tóxica $(0,09 \pm 0,08 \mathrm{mg} / \mathrm{L})$, nitrito $(0,12 \pm 0,13 \mathrm{mg} / \mathrm{L})$ e dureza total $(43,45 \mathrm{ppm})$ mantiveram-se dentro dos padrões aceitáveis para a espécie, de acordo com Arana (2004), o que sugere a não influência dessas variáveis sobre os resultados obtidos no experimento.

Os dados do desempenho zootécnico dos alevinos de tilápia do Nilo estão descritos na Tabela 2. Verificou-se que a inclusão de $5 \%$ de FFMD influenciou no peso e na taxa de crescimento específico dos animais. As análises de CMR, CAA, TEP, CP, CT, CCAB, ALT, IPERF e ICAB não apresentaram diferença.

Tabela 2. Desempenho de alevinos de tilápia do Nilo alimentados com diferentes níveis de FFMD na ração

\begin{tabular}{lcccccccc} 
& \multicolumn{3}{c}{ Níveis de FFMD } & & & & \\
& $0 \%$ & $5 \%$ & $10 \%$ & $15 \%$ & CV $(\%)$ & Teste F & Regressão & $\mathrm{R}^{2}$ \\
\cline { 2 - 5 } PI $(\mathrm{g})$ & 1,37 & 1,37 & 1,37 & 1,36 & 0,64 & $\mathrm{Ns}$ & $\mathrm{Y}=1,37$ & - \\
PF $(\mathrm{g})$ & 5,19 & 4,99 & 4,63 & 4,21 & 10,64 & 3,61 & $\mathrm{Y}=-6,6 \mathrm{x}+5,25$ & 0,97 \\
GP $(\mathrm{g})$ & 2,80 & 2,65 & 2,38 & 2,09 & 14,68 & 3,60 & $\mathrm{Y}=-4,8 \mathrm{x}+2,84$ & 0,98 \\
CMR $(\mathrm{g})$ & 5,98 & 5,19 & 5,57 & 5,24 & 12,55 & $\mathrm{Ns}$ & $\mathrm{Y}=21,98$ & - \\
CAA & 2,20 & 1,95 & 2,35 & 2,57 & 19,08 & $\mathrm{Ns}$ & $\mathrm{Y}=2,27$ & - \\
TEP & 1,46 & 1,67 & 1,34 & 1,26 & 19,94 & $\mathrm{Ns}$ & $\mathrm{Y}=1,43$ & - \\
CP $(\mathrm{cm})$ & 5,19 & 5,17 & 5,00 & 5,09 & 4,06 & $\mathrm{Ns}$ & $\mathrm{Y}=5,11$ & - \\
CT $(\mathrm{cm})$ & 6,59 & 6,31 & 6,24 & 6,36 & 3,68 & $\mathrm{Ns}$ & $\mathrm{Y}=6,37$ & - \\
CCAB $(\mathrm{cm})$ & 1,68 & 1,61 & 1,60 & 1,64 & 4,96 & $\mathrm{Ns}$ & $\mathrm{Y}=1,63$ & - \\
ALT $(\mathrm{cm})$ & 1,71 & 1,67 & 1,68 & 1,56 & 5,61 & $\mathrm{Ns}$ & $\mathrm{Y}=1,65$ & - \\
IPERF & 3,87 & 3,78 & 3,73 & 3,75 & 7,69 & $\mathrm{Ns}$ & $\mathrm{Y}=3,78$ & - \\
ICAB & 3,93 & 3,94 & 3,92 & 3,87 & 4,59 & $\mathrm{Ns}$ & $\mathrm{Y}=3,91$ & - \\
FC & 0,018 & 0,020 & 0,019 & 0,016 & 8,08 & 4,22 & $\mathrm{Y}=-2,72 \mathrm{x}+2,684$ & 0,95 \\
TCE $(\%)$ & 2,65 & 2,59 & 2,43 & 2,25 & 8,63 & 3,53 & $\mathrm{Y}=-0,5 \mathrm{x}^{2}+0,061 \mathrm{x}+$ & 0,99 \\
& & & & & & & 0,0181 & \\
\hline
\end{tabular}

FFMD - Farinha da folha de mandioca desidratada; Ns - não significativo; peso inicial (PI); peso final (PF); ganho de peso (GP); consumo médio de ração (CMR); conversão alimentar aparente (CAA); taxa de eficiência proteica (TEP); comprimento padrão (CP); comprimento total (CT); comprimento cabeça (CCAB); altura (ATL); índice de perfil (IPERF); índice de cabeça (ICAB); fator de condição (FC); taxa de crescimento específico (TCE).

O peso final e o ganho de peso dos animais experimentais estiveram próximos aos valores obtidos no controle; no entanto, a CAA apresentou melhores resultados quando houve a inclusão de 5\% de FFMD.

Resultados similares quanto ao desempenho de crescimento foram observados nos estudos de Bohnenberger et al. (2010) com larvas e juvenis de tilápia do Nilo e inclusão de $20 \%$ de concentrado proteico da folha de mandioca na ração, e por Hisano et al., (2011) com 6\% da parte aérea da mandioca em substituição ao farelo de soja.
Dessa maneira, verifica-se que a tilápia do Nilo tanto na fase larval como juvenil tem bom aproveitamento alimentar quando se utilizam as partes aéreas da folha de mandioca como ingrediente na ração. Isso pode estar relacionado com o teor de proteína bruta (PB) existente nas folhas da mandioca (Santos et al., 2009a).

Estudos anteriores demonstraram que o tambaqui (Colossoma macropomum) tem crescimento satisfatório quando alimentado com $15 \%$ de farinha de feno da mandioca incluídos na ração (Pontes et al., 2006). Espécies de peixes com hábito alimentar onívoro geralmente apresentam 
bom aproveitamento nutricional quando alimentados com ingredientes derivados de vegetais contendo em sua composição altos teores de proteínas, carboidratos e lipídios, conforme verificado em estudos anteriores (Hisano et al., 2008; Hisano et al., 2011; Souza et al., 2013; Bezerra et al., 2014).

Ocorreu um decréscimo linear para as variáveis peso final, ganho de peso e taxa de crescimento específico conforme houve o acréscimo do teor de farinha de folha de mandioca desidratada acima de $5 \%$ de inclusão $(\mathrm{P}<0,05)$.

O resultado encontrado no presente experimento é reforçado por $\mathrm{Ng}$ e Wee (1989); quando trabalhando com níveis de inclusão de 20, 40, 60 e $100 \%$ da farinha da folha da mandioca, verificaram uma redução linear no desempenho de tilápias do Nilo à medida que aumentava o nível de inclusão. Possivelmente, esse fato esteja relacionado à redução da palatabilidade da parte aérea da mandioca, uma vez que o incremento do nível percentual de inclusão da FFMD ocasionou redução no ganho de peso e piora na conversão alimentar aparente dos indivíduos experimentais.

Entretanto, no presente estudo, os animais alimentados com $10 \%$ de FFMD obtiveram crescimento mais homogêneo em relação aos animais alimentados com $15 \%$ e $5 \%$ de FFMD (Fig.1).

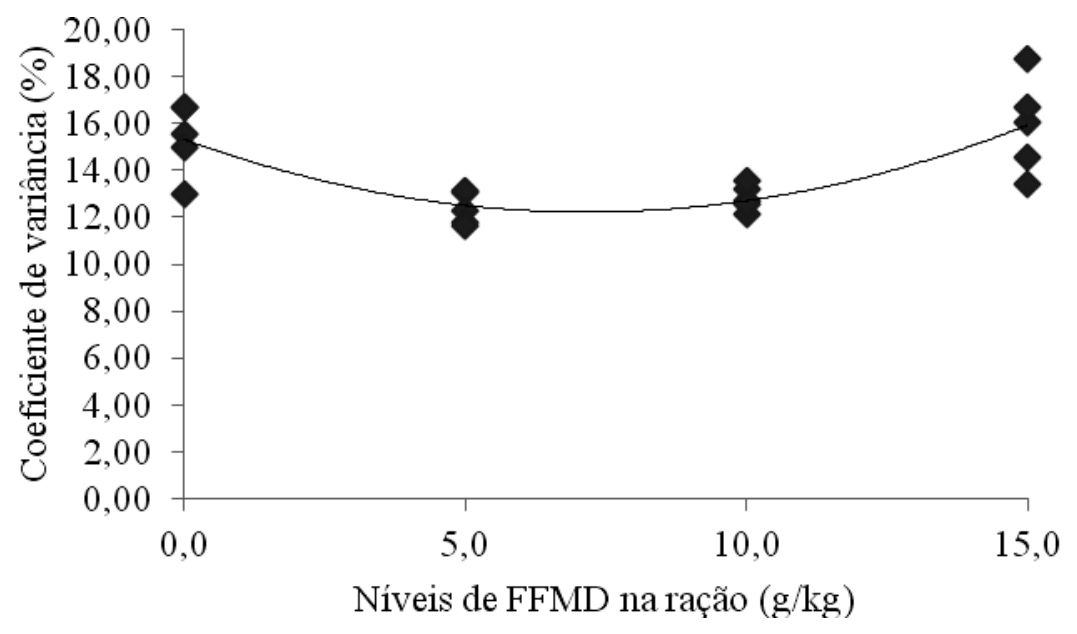

Figura 1. Gráfico da heterogeneidade do crescimento (CHET) de tilápia do Nilo alimentada com níveis de farinha de folha de mandioca desidratada na ração.

No entanto, os animais alimentados com $5 \%$ de FFMD apresentaram melhor desempenho de crescimento, ou seja, maior PF, GP, e melhor CAA, TCE, CMR. Essa afirmação abre precedente para a inclusão da FFMD em dietas para tilápias, mas de forma limitada, pois a espécie possui hábito territorialista, em que há uma hierarquia de dominação ocasionando heterogeneidade dos lotes (Corrêa et al., 2003); o aumento em $15 \%$ de inclusão poderá acarretar uma intensificação do comportamento agressivo da tilápia (Merighe et al., 2004). Assim, como observado no presente experimento, o maior ganho de peso dos animais foi obtido com os tratamentos contendo 5 e $10 \%$ de FFMD e, ao mesmo tempo, o menor índice de CHET também foi observado.

A viabilidade econômica da inclusão da folha de mandioca desidratada nas rações para alevinos de tilápia encontram-se na Tabela 3 . Foi verificada seguindo os preços (preço/kg) dos ingredientes utilizados na elaboração dos custos, que foram: milho grão ( $\mathrm{R} \$ 0,70)$, farelo de soja $45 \%(\mathrm{R} \$$ $0,85)$, folha de mandioca $(\mathrm{R} \$ 0,20)$, farelo de trigo ( $\mathrm{R} \$ 0,51)$, óleo de soja $(\mathrm{R} \$ 0,95)$, farinha de peixe $(\mathrm{R} \$ 0,95)$, calcário $(\mathrm{R} \$ 0,02)$, fosfato bicálcico ( $\mathrm{R} \$ 0,34)$, sal comum $(\mathrm{R} \$ 0,10)$, DLmetionina ( $\mathrm{R} \$ 3,50)$, premix mineral $\mathrm{e}$ vitamínico ( $\mathrm{R} \$ 3,00)$. Os preços dos ingredientes foram cotados durante o período experimental. 
Tabela 3. Custo da ração por quilograma (CRação), custo médio em ração por quilograma de peso vivo ganho (CMR), índice de custo (IC) e índice de eficiência econômica (IEE) de juvenis de tilápia do Nilo de acordo com os níveis de inclusão da folha de mandioca desidratada na ração

\begin{tabular}{|c|c|c|c|c|}
\hline \multirow[b]{2}{*}{ Variáveis } & \multicolumn{4}{|c|}{ Níveis de inclusão na ração } \\
\hline & $(0 \% \text { FFMD })^{1}$ & $(5 \% \text { FFMD })^{2}$ & $(10 \% \text { FFMD })^{3}$ & $(15 \% \text { FFMD })^{4}$ \\
\hline CRação (R\$/kg) & 0,804 & 0,780 & 0,738 & 0,720 \\
\hline $\mathrm{CMR}(\mathrm{R} \$ / \mathrm{kg} \mathrm{PVG})$ & 1,72 & 1,52 & 1,72 & 1,80 \\
\hline IC & 113,16 & 100 & 113,16 & 118,42 \\
\hline IEE & 88,37 & 100 & 88,37 & 84,44 \\
\hline
\end{tabular}

${ }^{1}-0 \%$ de inclusão da farinha da folha de mandioca desidratada.

${ }^{2}-5 \%$ de inclusão da farinha da folha de mandioca desidratada.

$3-10 \%$ de inclusão da farinha da folha de mandioca desidratada.

${ }^{4}-15 \%$ de inclusão da farinha da folha de mandioca desidratada.

A ração com a inclusão de 5\% de FFMD foi a que apresentou a melhor viabilidade econômica, nas condições estudadas. Esta foi utilizada de forma comparativa para os cálculos de índices de custo (IC) e índice de eficiência econômica (IEE). O tratamento com rações contendo $10 \%$ de FFMD apresentaram um custo médio semelhante ao tratamento controle ( $0 \%$ FFMD). Desse modo, seu uso também poderá ser indicado, desde que haja maior disponibilidade e facilidade de obtenção desse alimento.

A busca pela utilização de alimentos alternativos regionais tem o intuito de melhorar a eficiência na utilização da produção, conforme estudos já realizados (Nagae et al., 2001; Santos et al., 2009b; Bezerra et al., 2014). Além disso, a minimização dos custos com as rações é almejada incessantemente. De acordo com Meurer et al. (2000), o estudo dos alimentos alternativos procura dar subsídios para a produção de rações mais baratas e de mesma qualidade nutricional, proporcionando desempenho produtivo equivalente àquelas formuladas com alimentos convencionais.
Para o peixe piavuçu (Leporinus macrocephalus), avaliaram-se níveis de inclusão do triticale $(0 ; 5,14 ; 10,29 ; 15,43$ e $25,72 \%)$ para alevinos de $1,70 \mathrm{~g}$, demonstrando que não houve diferenças sobre a viabilidade econômica e sobrevivência entre os tratamentos, recomendando assim a inclusão de até $25,72 \%$ de triticale em dietas para piavuçu; ressaltando, porém, que a sua utilização dependerá da disponibilidade e preço no mercado (Nagae et al., 2001).

A FFMD é considerada na região Nordeste um ingrediente amplamente disponível para o uso em rações para peixes, com possibilidades de ampliação na sua exploração para fabricação de produtos direcionados à alimentação animal, minimizando os custos de produção. De acordo com o presente estudo, a utilização de $5 \%$ de FFMD em rações é viável economicamente nos cultivos de tilápia.

Os resultados dos parâmetros físiológicos dos animais alimentados com FFMD na ração estão expressos na Tabela 4.

Tabela 4. Parâmetros fisiológicos de alevinos de tilápia do Nilo alimentados com os diferentes níveis de FFMD na ração

\begin{tabular}{ccccccccc}
\hline & \multicolumn{3}{c}{ Níveis de FFMD } & & & \\
\cline { 2 - 4 } Variáveis & 0,0 & $5,0 \%$ & $10,0 \%$ & $15,0 \%$ & CV (\%) & Teste F & Regressão \\
\hline IDS (\%) & 0,73 & 0,50 & 0,85 & 0,76 & 12,76 & Ns & $\mathrm{Y}=7,63$ \\
IHS (\%) & 7,95 & 6,85 & 7,40 & 8,32 & 41,09 & Ns & $\mathrm{Y}=0,71$ \\
P Fig (g) & 0,04 & 0,03 & 0,04 & 0,03 & 33,15 & Ns & $\mathrm{Y}=0,03$ \\
P TGI (g) & 0,42 & 0,34 & 0,35 & 0,35 & 19,17 & Ns & $\mathrm{Y}=0,36$
\end{tabular}

Índice digestivossomático (IDS); índice hepatossomático (IHS); peso do fígado (P Fig); peso do trato gastrointestinal (P TGI). 
Não houve diferenças entre os resultados obtidos nos parâmetros de desempenho fisiológico, como índice digestivossomático, índice hepatossomático, peso do fígado e peso do trato gastrointestinal, indicando que a FFMD presente nos diferentes tratamentos em nada influenciou nesses índices. Segundo Lizama et al. (2007), as relações entre os órgãos, como fígado, rim, olho, baço e gônadas, ou medidas de comprimento e peso, bem como o estudo do fator de condição dos peixes, é uma importante ferramenta para o desempenho zootécnico e bem-estar animal, já que os órgãos do trato gastrointestinal e fígado são sensíveis ao tipo de alimentação devido às transformações metabólicas e absorção dos nutrientes.

Por outro lado, possíveis resultados desfavoráveis do uso da folha da mandioca no presente estudo pode ser devido aos compostos glicídeos cianogênicos (cianeto), que são compostos antinutricionais termolábeis inibidores de proteases e lecitinas, antivitaminas e que interferem no uso do alimento (Corrêa et al., 2004; Melo et al., 2007), afetando a saúde em peixes. O teor de cianeto na mandioca, dependendo da variedade, pode ser até seis vezes maior nas folhas em comparação às raízes, podendo ser altamente tóxica para os animais, além do alto teor de fibra que pode limitar a sua inclusão e piorar o aproveitamento de nutrientes. Tal fato foi relatado por $\mathrm{Ng}$ e Wee (1989), ao avaliarem a digestibilidade da proteína bruta e matéria seca de rações que substituíram em até $100 \%$ da proteína da farinha de peixe pela farinha de folha de mandioca. Os autores concluíram que, à medida que se aumentou a inclusão de folha de mandioca na ração, houve piora nos coeficientes de digestibilidade aparente da proteína da ração e na sua digestibilidade total.

Outro fator a ser considerado é o processamento e a forma de obtenção da FFMD; segundo Leonel (2001), o sistema de corte e a porção da parte aérea utilizada podem influenciar nos resultados, pois a composição química e o valor nutritivo podem ser afetados por esses fatores.

A substituição de determinados produtos e subprodutos da agroindústria, empregados como ingredientes das dietas dos peixes, por produtos sucedâneos, tem se apresentado como prática econômica alternativa. E de tal modo que a viabilidade econômica e custo/benefício da utilização da FFMD para tilápia do Nilo devem ser levados em consideração no momento da formulação das rações. Apesar de a FFMD não ter produzido efeito linear positivo, o seu uso deve ser incentivado dependendo da disponibilidade na região e do custo desse alimento em comparação com as outras fontes de nutrientes.

De modo geral, a inclusão da folha de mandioca desidratada na ração para alevinos de tilápia do Nilo interferiu negativamente no desempenho produtivo dos animais. Entretanto, a utilização de $5 \%$ da farinha de folha de mandioca na ração mostrou-se economicamente viável.

\section{CONCLUSÃO}

Recomenda-se o uso de 5\% de farinha de folha de mandioca desidratada em rações completas para alevinos de tilápia do Nilo.

\section{REFERÊNCIAS}

ARANA, L.V. Princípios químicos de qualidade de água em aquicultura: uma revisão para peixes e camarões. 2.ed. Florianópolis: UFSC, 2004. 231p.

BEZERRA, S.K.; SOUZA, R.C.; MELO, J.F.B.; CAMPECHE, D.F.B. Crescimento de tambaqui alimentado com diferentes níveis de farinha de manga e proteína na ração. Arch. Zootec., v.63, p.587-598, 2014.

BOHNENBERGER, L.; GOMES, S.D.; COELHO, S.R.M. et al. Concentrado proteico de folhas de mandioca na alimentação de tilápia do nilo na fase de reversão sexual. Rev. Bras. Zootec., v.39, p.1169-1174, 2010.

BOLETIM ESTATÍSTICO DA PESCA E AQUICULTURA 2011. Brasília: MPA, 2011. 60p.

BOSCOLO, W.R.; HAYASHI, C.; MEURER, F. Farinha de varredura de mandioca (Manihot esculenta) na alimentação de alevinos de tilápia do Nilo (Oreochromis niloticus). Rev. Bras. Zootec., v.31, p.546-551, 2002.

CORRÊA, A.D.; SANTOS, S.R.; ABREU, C.M.P. et al. Remoção de polifenóis da farinha de folhas de mandioca. Ciênc. Tecnol. Aliment., v.24, p.159164, 2004.

CORRÊA， S.A.; FERNANDES, M.O.; ISEKI, K.K. et al. Effect of the establishment of dominance relationships on cortisol and other 
metabolic parameters in Nile tilapia (Oreochromis niloticus). Braz. J. Med. Biol. Res. v.36, p.17251731, 2003.

HISANO, H.; BARROS, M.B.; PEZZATO, L.E. Desempenho produtivo de tilápias-do-Nilo alimentadas com rações contendo parte aérea de mandioca. Cuiabá: Embrapa/Agropecuária Oeste, 2011 19p. (Boletim de Pesquisa e Desennvolvimento, 59).

HISANO, H.; MARUAYAMA, M.R.; ISHIKAWA, M.M. et al. Potencial da utilização da mandioca na alimentação de peixes. Cuiabá: Embrapa/Agropecuária Oeste, 2008, 29p.

LACERDA, C.H.F.; HAYASHI, C.; SOARES, C.M. et al. Farelo de mandioca (Manihot esculenta) grants em substituição ao milho (Zea mays L.) em rações para alevinos de carpa-capim (Ctenopharyngodon idella). Acta Sci. Anim. Sci., v.27, p.241-245, 2005.

LEONEL, M. Uso dos subprodutos da industrialização da mandioca na alimentação animal. In: CEREDA, M.P. (Coord.) Manejo, uso e tratamento de subprodutos da industrialização da mandioca. [s.1.]: Fundação Cargill, 2001. p.229239.

LIZAMA, M.A.P.; TAKEMOTO, R.M.; RANZANI-PAIVA, M.J.T. et al. Relação parasitohospedeiro em peixes de pisciculturas da região de hospedeiro em peixes de pisciculturas da região de Assis, Estado de São Paulo, Brasil. Oreochromis niloticus (Linnaeus, 1757). Acta Sci. Biol. Sci., v.29, p.223-231, 2007.

MELO, D.S.; CORRÊA, A.D.; MARCOS, F.C.A. et al. Efeitos da farinha de folhas de mandioca sobre a peroxidação lipídica, o perfil lipídico sanguíneo e o peso do fígado de ratos. Ciênc. Agrotec., v.31, p.420-428. 2007.

MERIGHE, G.K.F.M.; PEREIRA-DA-SILVA, E.M.; NEGRÃO, J.A.; RIBEIRO, S. Efeito da cor do ambiente sobre o estresse social em tilápias do Nilo (Oreochromis niloticus). Rev. Bras. Zootec.,v.33, p.828-837, 2004.
MEURER, F.; HAYASHI, C.; SOARES, C.M. et al. Utilização de levedura spray-dried na alimentação de alevinos de tilápia do Nilo (Oreochromis niloticus, L.). Acta Scient., v.22, p.479-484, 2000.

NAGAE, M.Y.; HAYASHI, C.; GALDIOLI, E. M. Inclusão do triticale em rações para alevinos de piavuçu, Leporinus macrocephalus (Garavello \& Britski, 1988). Acta Scient. Anim. Sci., v.23, p.849853, 2001.

NG, W.K.; WEE, K.L. The nutritive value of cassava leaf meal in pelleted feed for Nile tilapia. Aquaculture, v.83, p.45-58, 1989.

NUTRIENT requirements of fish. Washington, D.C.: National Washington Academy Press, 1993. 114p.

PONTES, E.C.; MIRANDA, E.C.; FRAGA, A.B. et al. Farinha do feno da folha de mandioca como constituinte de rações sobre o desempenho produtivo do tambaqui (Colossoma macropomum). In: REUNIÃO ANUAL DA SOCIEDADE BRASILEIRA DE ZOOTECNIA, 43， 2006, Paraíba. Anais... João Pessoa: SBZ, 2006. CDROM. (Resumo expandido).

SANTOS, E.L.; LUDKE, M.C.M.M.; BARBOSA, J.M. Níveis de farelo de coco em rações para alevinos de tilápia do Nilo. Rev. Bras. Saúde Prod. Animal, v.10, p.390-397. 2009b.

SANTOS, E.L.; LUDKE, M.C.M.M.; RAMOS, A.M.P. et al. Digestibildade de subprodutos da mandioca para a Tilápia do Nilo. R. Bras. Ciên. Agrár. v.4, p.358-362. 2009a.

SANTOS, E.L.; SILVA, F.C.B.; PONTES, E.C. et al. Resíduo do processamento do extrato de própolis vermelha em ração comercial para alevinos de Tilápia do Nilo (Oreochromis niloticus) Comunic. Scient., v.4, p.179-185. 2013.

SILVA, A.M.R.; BERTO, D.A.; LIMA, G.J.M.M. et al. Valor nutricional e viabilidade econômica de rações suplementadas com maltodextrina e acidificante para leitões desmamados. Rev. Bras. Zootec., v.37, p.286-295, 2008.

SOUZA, R.C.; MELO, J.F.B.; NOGUEIRA FILHO, R.M. et al. Influência da farinha de manga no crescimento e composição corporal da tilápia do Nilo. Arch. Zootec., v.62, p.217-225, 2013. 